\title{
Erratum to: Intraoperative Patterns of Gastric Microperfusion During Laparoscopic Sleeve Gastrectomy
}

\author{
Tarik Delko $^{1}$ - Henry Hoffmann ${ }^{1} \cdot$ Marko Kraljević $^{1} \cdot$ Raoul A. Droeser $^{1}$ • \\ Lincoln Rothwell ${ }^{2}$ - Daniel Oertli ${ }^{1}$ - Urs Zingg ${ }^{3}$
}

Published online: 23 March 2017

(C) Springer Science+Business Media New York 2017

Erratum to: OBES SURG

DOI 10.1007/s11695-016-2386-7

The spelling of author name Marko Kraljević was incorrect in the original article. It is correct here.

The online version of the original article can be found at http: \ldx.doi. $\operatorname{org} \backslash 10.1007 / \mathrm{s} 11695-016-2386-7$.

Tarik Delko

tarik.delko@gmail.com

1 Department of General and Visceral Surgery, University Hospital Basel, Spitalstrasse 21, 4031 Basel, Switzerland

2 Department of Surgery, Ipswich General Hospital, Ipswich, QLD, Australia

3 Department of Surgery, Limmattal Hospital, Zurich-Schlieren, Switzerland 